\title{
Quantized gravito-magnetic charges from WIMT: cosmological consequences
}

\author{
1,2 Jesús Martín Romero*, ${ }^{1,2}$ Mauricio Bellini ${ }^{\dagger}$ \\ ${ }^{1}$ Departamento de Física, Facultad de Ciencias Exactas y Naturales, \\ Universidad Nacional de Mar del Plata, \\ Funes 3350, C.P. 7600, Mar del Plata, Argentina. \\ ${ }^{2}$ Instituto de Investigaciones Físicas de Mar del Plata (IFIMAR), \\ Consejo Nacional de Investigaciones Científicas y Técnicas (CONICET), Argentina.
}

\begin{abstract}
Using the formalism of Weitzenböck induced matter theory (WIMT) we calculate the gravitomagnetic charge on a topological string which is induced through a foliation on a five-dimensional (5D) gravito-electromagnetic vacuum defined on a 5D Ricci-flat metric, which produces a symmetry breaking on an axis. We obtain the resonant result that the quantized charges are induced on the effective four-dimensional hypersurface. This quantization describes the behavior of a test gravitoelectric charge in the vicinity of a point gravito-magnetic monopole, both geometrically induced from a $5 \mathrm{D}$ vacuum. We demonstrate how gravito-magnetic monopoles would decrease exponentially during the inflationary expansion of the universe.
\end{abstract}

\footnotetext{
* E-mail address: jesusromero@conicet.gov.ar

$\dagger$ E-mail address: mbellini@mdp.edu.ar
} 


\section{INTRODUCTION AND MOTIVATION}

For some decades, topological defects have been a very important subject of research[1]. The existence of stable topological defect solutions was established in realistic renormalizable theories and many developments were required in the understanding of phase transitions. In this framework defect solutions were discovered in Higgs and Yang-Mills theories, the Nielsen-Olesen vortex-line[2] and the t'Hooft-Polyakov magnetic monopole[3]. For instance, strings quantization has been studied in the framework of a $A d S_{5} \times S^{5}[4]$. The study of the cosmological implications of topological defects has become an area of sustained interest [5]. In this context cosmic strings may provide a viable spectrum of galaxy formation[6, 7]. Another topic of great interest is the study of the evolution of magnetic monopoles in the universe. The Big Bang cosmology predicts that a very large number of heavy, stable "magnetic monopoles" should have been produced in the early universe. However, magnetic monopoles have never been observed, so if they exist at all, they are much rarer than the Big Bang theory predicts. Inflationary cosmology resolves this problem[8]. During inflation, the density of monopoles drops exponentially, so their abundance drops to undetectable levels. Monopoles are just created before (or during) inflation, so that the rapid accelerated expansion thereafter dilutes their density to very low levels. Monopoles are created, they are created at a density of order of one per Hubble volume, which means that there is one (or fewer) in each observable Universe, so monopoles are separated by a distances of the order of the Hubble length (or larger).

However, there is a more interesting possibility that arises from a theory that unifies conceptually electrodynamics with a theory of gravity. Gravito-electrodynamics was first outlined in 2006 in a cosmological context as gravito-electromagnetic inflation[9] with the aim of describing in an unified manner both, primordial gravitational and electromagnetic

effects in the early inflationary universe[10, 11]. This is a gravito-electrodynamic formalism constructed with a penta-vector with components $A^{b}$ that can be applied to any physical system in the framework of the induced matter theory (IMT)[12]. This theory is based on the assumption that ordinary matter and physical fields that we can observe in our four-dimensional (4D) universe can be geometrically induced from a five-dimensional (5D) Ricci-flat metric with a space-like noncompact extra dimension on which we define a physical vacuum. Because we shall be dealing with a penta-vector $A$ in a relativistic framework, 
it will be natural to consider a 5D relativistic theory. This theory is based on the assumption that ordinary matter and physical fields that we can observe in our 4D universe can be geometrically induced from a 5D Ricci-flat metric with a space-like noncompact extra dimension on which we define a physical vacuum[12]. The Campbell-Magaard theorem[1316] serves as a ladder to go between manifolds whose dimensionality differs by one. This theorem, which is valid in any number of dimensions, implies that every solution of the 4D Einstein equations with arbitrary energy momentum tensor can be embedded, at least locally, in a solution of the 5D Einstein field equations in vacuum. Because of this, the stress-energy may be a 4D manifestation of the embedding geometry. An extension of the IMT was realized recently using the Weitzenböck induced matter theory (WIMT)[17]. This approach makes possible a geometrical representation of a $5 \mathrm{D}$ vacuum (with a zero curvature in the Weitzenböck representation), of a nonzero curvature tensor (in the sense of the Levi-Civita representation). The WIMT formalism was introduced with the intent to generalize the foliation's method in the IMT of gravity, because nonstatic foliations result in very difficult calculations. With the WIMT formalism, one can replace a dynamic foliation over a 5D Ricci-flat space (in a Levi-Civita representation), by a static foliation from a 5D curved space (in a Levi-Civita representation), on which one defines a 5D vacuum from the point of view of a Weitzenböck representation[18]. Once done the foliation we can go to the representation of Levi-Civita. This procedure provides a huge versatility to make static foliations from $5 \mathrm{D}$ curved manifolds to obtain arbitrary $4 \mathrm{D}$ hypersurfaces.

In this work we shall consider a $5 \mathrm{D}$ space-time described by the metric $g_{a b}=e_{a}^{A} e_{b}^{B} \eta_{A B}{ }^{1}$ in a $5 \mathrm{D}$ Weitzenböck vacuum with the aim of quantizing the gravito-magnetic charge on a topological string which is induced through a foliation on a 5D Ricci-flat metric. We shall use the Weitzenböck representation because it makes possible the use of the Campbell-Magaard theorem on a Weitzenböck-flat 5D space. Such a space is Weitzenböck-flat in the sense that the Riemann tensor constructed through this kind of connections is null: ${ }^{(W)} R_{b c d}^{a}=0$. However, it cannot be Riemann-flat with respect to the Levi-Civita connections: ${ }^{(l c)} R_{b c d}^{a} \neq 0$.

The paper is organized as follows: in Sect. II we review the Weitzenböck representation of space and the gravito-electromagnetic theory in the framework of WIMT. In Sect. III we explore the evolution of gravito-magnetic charges in an expanding universe and we calculate

\footnotetext{
${ }^{1}$ We shall denote by $\eta_{A B}$ the tensor metric in a 5D Minskowsky spacetime.
} 
quantizate this charge in the Dirac's sense. Finally, in Sect. IV we develop some final comments.

\section{WEITZENBÖCK REPRESENTATION OF SPACE AND GRAVITO- ELECTROMAGNETIC FIELDS FROM WIMT}

The Riemann tensor written with the Weitzenböck representation for the space-time characterized by the metric $g_{a b}$, is given by

$$
{ }^{(W)} R_{b c d}^{a}=\vec{e}_{b}\left({ }^{(W)} \Gamma_{d c}^{a}\right)-\vec{e}_{c}\left({ }^{(W)} \Gamma_{d b}^{a}\right)+{ }^{(W)} \Gamma_{d c}^{n}{ }^{(W)} \Gamma_{n b}^{a}-{ }^{(W)} \Gamma_{d b}^{n}{ }^{(W)} \Gamma_{n c}^{a}-C_{c b}^{n}{ }^{(W)} \Gamma_{d n}^{a}=0
$$

where ${ }^{(W)} \Gamma_{b c}^{a}$ are the Weitzenböck connections and $C_{b c}^{a}$ are the coefficients of structure of the base in which we write $g_{a b}$. They can be expressed through $C_{b c}^{a}=\bar{e}_{N}^{a} \vec{e}_{c}\left(e_{b}^{N}\right)-$ $\bar{e}_{N}^{a} \overrightarrow{e_{b}}\left(e_{c}^{N}\right)={ }^{(W)} \Gamma_{b c}^{a}-{ }^{(W)} \Gamma_{c b}^{a}$. When the absence of structure of the Minkowsky spacetime $[\eta]_{A B}=\operatorname{diag}[1,-1,-1,-1,-1]$ makes the Weitzenböck torsion null, both representations (Levi-Civita and Weitzenböck), are related by the expression

$$
{ }^{(W)} \Gamma_{b c}^{a}={ }^{(l c)} \Gamma_{b c}^{a}-{ }^{(W)} K_{b c}^{a},
$$

where, in the absence of no-metricity $g_{a b ; c}=0^{2}$, the Weitzenböck contortion ${ }^{(W)} K_{b c}^{a}$ being given by the Weitzenböck torsion ${ }^{(W)} T_{b c}^{a}$

$$
{ }^{(W)} K_{b c}^{a}=\frac{g^{m a}}{2}\left\{{ }^{(W)} T_{c m}^{n} g_{b n}+{ }^{(W)} T_{b m}^{n} g_{n c}-{ }^{(W)} T_{c b}^{n} g_{n m}\right\} .
$$

Equation (1) shows that one can pass from a Weitzenböck to a Levi-Civita representation once the contortion ${ }^{(W)} K_{b c}^{a}$ is known.

We shall consider the conditions by which we can induce curvature and currents by means of WIMT, on a 5D space-time represented by Cartesian coordinates. The action for the gravito-electromagnetic fields in a $5 \mathrm{D}$ vacuum can be written in terms of the $F_{A B}$ tensor components or in terms of the dual tensors $\mathcal{F}_{A B C}$

$$
\begin{aligned}
\mathcal{S} & =\int d^{5} x \sqrt{|\eta|}\left[\frac{R}{16 \pi G}-\frac{1}{4} F_{A B} F^{A B}\right] \\
& =\int d^{5} x \sqrt{|\eta|}\left[\frac{R}{16 \pi G}-\frac{k}{4} \mathcal{F}_{A B C} \mathcal{F}^{A B C}\right],
\end{aligned}
$$

\footnotetext{
${ }^{2}$ We can assure it because we are starting the transformation from a Minkowsky metric with coefficients $\eta_{A B}$.
} 
where $\frac{1}{3 !} \mathcal{F}_{A B C} \mathcal{F}^{A B C}=\frac{1}{3 ! 4} \varepsilon_{A B C D E} \varepsilon^{A B C N M} F^{D E} F_{N M}=F^{N M} F_{N M}$, and $\varepsilon_{A B C D E} \varepsilon^{A B C N M}=$ $3 ! 2 !\left(\delta_{D}^{N} \delta_{E}^{M}-\delta_{E}^{N} \delta_{D}^{M}\right)$, so that when $k=\frac{1}{3 !}$, we obtain that both actions describe the same physical system[19]. In our case, when we use the Lorentz gauge, we deal with a 5D vacuum, so that $R=0$. The gravito-electromagnetic dynamics, after taking into account the Lorentz gauge: $A_{; B}^{B}=0$ in the action (3), are

$$
\square A^{K}=\eta^{B C} A_{; B C}^{K}=0 .
$$

The gravito-magnetic currents come from the solutions for the fields (4). The last equations are compatible with a current that has its source in

$$
F_{; B}^{N B}=-\eta^{A N}\left[A^{M} R_{A B M}^{B}+A_{; M}^{B} T_{B A}^{M}\right]
$$

Using the expression ${ }^{(l c)} \Gamma_{B C}^{A}={ }^{(W)} \Gamma_{B C}^{A}+K_{B C}^{A}$ it is possible to obtain the following expression between both Faraday tensors:

$$
{ }^{(l c)} F^{N B}={ }^{(W)} F^{N B}+\eta^{R N} A^{P} K_{P R}^{B}-\eta^{R B} A^{P} K_{P R}^{N} .
$$

The Weitzenböck currents are related to the Levi-Civita ones by the expression

$$
{ }^{(l c)(m)} J_{A B}-{ }^{(W)(m)} J_{A B}=\frac{\sqrt{|\eta|}}{2} \varepsilon_{A B C D E} \frac{1}{4} M^{[C D E]},
$$

such that the antisymmetric source $M^{[C D E]}=\eta^{C F} \eta^{D G} \eta^{E H} M_{[F G H]}$ is given by the expression

$$
\begin{aligned}
M_{[F G H]} & \left.=\left(A_{M}{ }^{(W)} T_{[F G}^{M}\right) ; H\right]-2^{(W)} T_{[F H \mid}^{N}{ }^{(W)} T_{N \mid G]}^{M} A_{M}-2^{(W)} T_{[G H}^{N}{ }^{(W)} T_{F] N}^{M} A_{M} \\
& -{ }^{(W)} T_{[F H \mid}^{N} \vec{E}_{N}\left(A_{\mid G]}\right)+{ }^{(W)} T_{[G H \mid}^{N} \vec{E}_{N}\left(A_{\mid F]}\right)+{ }^{(W)} T_{[F H}^{N} \vec{E}_{G]}\left(A_{N}\right) \\
& -{ }^{(W)} T_{[G H}^{N} \vec{E}_{F]}\left(A_{N}\right) .
\end{aligned}
$$

Notice that the gauge condition in the Levi-Civita representation ${ }^{(l c)} A_{; N}^{N}=0$, it is preserved in the Weitzenböck one: ${ }^{(W)} A_{; n}^{n}=0$.

\section{QUANTIZED GRAVITO-MAGNETIC CHARGES IN AN EXPANDING UNI- VERSE}

To consider the evolution of a gravito-magnetic charge in an expanding universe (for instance, during the early inflationary universe), we shall consider the vielbein given by 
$E_{n}^{N}:=\operatorname{diag}(1, a(t), a(t), a(t), 1)$, defined with respect to the 5D Minkowsky space-time $\eta_{a b}=\operatorname{diag}[1,-1,-1,-1,-1]$, which is written in cartesian coordinates. Because we are in a comoving frame, the components $U^{B}$, of the penta-velocity will be described by $U \equiv(1,0,0,0,0)$. In this case the base of the tangent space $T_{p}(M)$ will be given by the elements $B=\left\{\frac{\vec{\partial}}{\partial t}, a(t) \frac{\vec{\partial}}{\partial x}, a(t) \frac{\vec{\partial}}{\partial y}, a(t) \frac{\vec{\partial}}{\partial z}, \frac{\vec{\partial}}{\partial l}\right\}_{p}$, where the relevant non-zero structure coefficients are $C_{i 0}^{i}=\dot{a}(t)$. The elements of the resulting covariant tensor metric are given by

$$
g_{a b}=\operatorname{diag}\left[1,-a^{2}(t),-a^{2}(t),-a^{2}(t),-1\right] .
$$

In order to illustrate the formalism, now we shall consider the case where the torsion is induced through the vielbein

$$
\bar{e}_{N=0}^{n=0}=1, \bar{e}_{N=1}^{n=1}=1, \bar{e}_{N=1}^{n=2}=\varepsilon \frac{\partial \phi(x, y)}{\partial x}, \bar{e}_{N=2}^{n=2}=1+\varepsilon \frac{\partial \phi(x, y)}{\partial y}, \bar{e}_{N=3}^{n=3}=1, \bar{e}_{N=4}^{n=4}=0
$$

This means that the effective 4D energy momentum tensor will be

$$
{ }^{(4 D)} T_{\mu}^{\nu}=\left.\bar{e}_{N}^{n=\nu} e_{n=\mu}^{M}{ }^{(5 D)} T_{M}^{N}\right|_{l=l_{0}} .
$$

Furthermore, the effective 4D tensor metric will be

$$
[g]_{\alpha \beta}=\left(\begin{array}{cccc}
1 & 0 & 0 & 0 \\
0 & -a^{2}(t) & 0 & 0 \\
0 & 0 & \left(-1+\varepsilon^{2}\left(\left(\frac{\partial \phi(x, y)}{\partial x}\right)^{2}-\frac{2}{\varepsilon} \frac{\partial \phi(x, y)}{\partial y}-\left(\frac{\partial \phi(x, y)}{\partial y}\right)^{2}\right)\right) a^{2}(t) & 0 \\
0 & 0 & 0 & -a^{2}(t)
\end{array}\right),
$$

where $\varepsilon$ is an arbitrary small parameter. In order to make coordinated the resulting base of the space-time, we shall make the choice $\phi(x, y)=\arctan (y / x)$. The resulting effective 4D space-time $g_{\alpha \beta}$ will be twisted

$$
g_{\alpha \beta}=\left.e_{\alpha}^{A} e_{\beta}^{B} g_{A B}\right|_{l=l_{0}},
$$

where the vielbein $e_{\alpha}^{A}$ are the inverse of those in (10): $e_{\alpha}^{A} \bar{e}_{A}^{\beta}=\delta_{\alpha}^{\beta}$. The base in terms we write the metric $g_{\alpha \beta}$ in (12) will be free of structure, but with nonzero torsion: ${ }^{(W)} T_{\mu \nu}^{2}=$ $\bar{e}_{N=\lambda}^{n=2}{ }^{(W)} T_{\mu \nu}^{\lambda}=-\varepsilon\left(\partial_{\mu} \partial_{\nu}-\partial_{\nu} \partial_{\mu}\right) \phi(x, y)$. Using the Stokes theorem on the $x y$ plane, one can see that this calculation is compatible with a Weitzenböck torsion given by ${ }^{(W)} T_{12}^{2}=$ $-2 \varepsilon \pi \delta^{(2)}(x, y)$. Hence, the torsion will be on a string that is located on the $z$ axis. In this way, although the sources are null on the 5D space-time, the foliation drives a symmetry 
breaking capable of inducing an effective torsion that generates gravito-magnetic monopoles with a volumetric density of charge $\rho_{m}={ }^{(4 D)(l c)(m)} J_{0}=\left.{ }^{(5 D)}\left({ }^{(l c)(m)} J_{A B} U^{B}\right) e_{0}^{A}\right|_{l=l_{0}}$, such that the gravito-magnetic density of charge $\rho_{m}$ can be obtained using the tensor current $(l c)(m) J_{A B}$ in (7)

$$
{ }^{(l c)(m)} J_{A B}={ }^{(W)(m)} J_{A B}+\frac{\sqrt{|\eta|}}{2} \varepsilon_{A B C D E} \frac{1}{4} M^{[C D E]},
$$

$M^{[C D E]}$ being given by (요). Hence, one obtains

$$
\rho_{m}=-4 A_{2,3} \varepsilon \pi \delta^{(2)}(x, y) g^{22}
$$

Notice that this result is dependent of the choice for the vielbein (10), which incorporates a non-holonomic transformation such that $y \rightarrow y^{\prime}=y+\varepsilon \phi(x, y)$ represents a topological defect similar to a dislocation[20].

Finally, in order to close the calculation we shall study the quantization of gravitomagnetic and gravito-electric charges. In this sense we follow the Vilenkin and Shellard argument[21]. This leads to a result for the amplitude of a particle to go around a closed path, $\mathcal{A}$. The following proportionality relation is set as

$$
\mathcal{A} \sim e^{i Q_{g m} \oint_{\Gamma} \mathbf{A} \cdot \mathbf{d x}}=e^{i Q_{g m} \int_{\Sigma} \mathbf{B} \cdot \mathbf{d s}},
$$

where the surface $\Sigma$ is bounded by a closed path called $\Gamma$ and $Q_{g m}$ is the gravito-magnetic charge. In this case we apply (15) to gravito-magnetic and the gravito-electric charges, $Q_{g m}$ and $Q_{g e}$, and choose $\Gamma$ as a circumference of radius $\rho$ centered on the $\mathbf{z}$-axis. Furthermore, $\Sigma$ is considered as a cylindrical surface bounded by $\Gamma$. All the calculation was done in order to quantize the charges on the effective 4D hypersurface, such that the gravito-magnetic field is reduced to $\mathbf{B}=\frac{Q_{g m}}{2 \pi \rho^{2}} \hat{\rho}$ and all the effective tensors must take a form analogous to (11). Taking into account the most basic solutions of (4), associated with the zero mode of the field and the symmetry of problem, we obtain

$$
\oint_{\Gamma} \mathbf{A} \cdot \mathbf{d} \theta=\left[\frac{2(1+\varepsilon) \varepsilon}{a(t)} e^{-K_{l} \frac{l-l_{0}}{l_{0}}} \int d \phi\right]_{l=l_{0}}=\frac{4 \pi \varepsilon(1+\varepsilon)}{a(t)},
$$

because $\int d \phi=2 \pi$ over a full turn. The gravito-magnetic charge fulfills the expression

$$
Q_{g m}=m\left(\frac{\varepsilon(1+\varepsilon)}{a(t)}\right),
$$

where $m$ is an integer number, and (17) is qualitatively compatible with (14). In the same way, we can work the gravito-electric induced charge

$$
Q_{g e}=n\left(\frac{a(t)}{\varepsilon(1+\varepsilon)}\right)
$$


where $n$ is an integer. Hence, the product of both charges complies with Dirac's law of quantization

$$
Q_{g e} Q_{g m}=(m n) .
$$

This result is very important and shows how the product $Q_{g e} Q_{g m}$, results in an invariant on an expanding universe. If we take in mind, for instance, an early inflationary universe with a scale factor $a(t)=a_{0} e^{H_{0} t}$, it is easy to see that gravito-magnetic charges $Q_{g m}$ will be exponentially decreasing during the inflationary expansion of the universe ${ }^{3}$, meanwhile gravito-electric charges $Q_{g e}$ will be constant for a comoving observer.

\section{REMARKS}

We have used WIMT in the particular example in which the foliation reveals a topological defect in the effective 4D arrival space-time. In the example we have obtained the presence of localized gravito-magnetic charges distributed along the $\mathbf{z}$-axis. The gravito-magnetic charges appear to be associated with the Weitzenböck torsion of space-time. This torsion is located on the topological defect and induced by a non-holonomic foliation. The gravitomagnetic charge distribution here obtained is expressed within the Levi-Civita derivative operator, so that it is a genuine gravito-magnetic charge distribution and was finally calculated in a Riemannian geometric construction (although its source lies in a Weitzenböck torsion). The quantization of charges was carried out in the effective $4 \mathrm{D}$ hypersurface. Hence, the Dirac quantization describes the behavior of a test gravito-electric charge in the vicinity of a point gravito-magnetic monopole, both geometrically induced.

A priori, in a STM theory, we can expect that the quantization of charges can take place in $4 \mathrm{D}$ space because the assumption of an empty 5D material space. Although in this case we chose to develop an effective quantization, a $5 \mathrm{D}$ quantization may be obtained at the higher space-time when ${ }^{(5 D)} R_{A B}=\lambda^{(5 D)} g_{A B} \neq 0$ and charges must exist. Then one would apply WIMT to obtain the effective $4 \mathrm{D}$ charges. The fact of using only the zero mode of the field for the effective quantization, in addition to providing operational simplicity, can be related to the fact that configuration of gravito-electric and gravito-magnetic charges are

\footnotetext{
${ }^{3}$ Of course one could mean in a collapsing pre-Big Bang scenario in which the scale factor is decreasing, so that the gravito-magnetic charges $Q_{g m}$ could be increased before the Big Bang.
} 
comoving/static in the example here studied. We can see how the gravito-magnetic charge decays rapidly with time, and therefore during the inflationary epoch they should disappear for a comoving observer, due to the accelerated expansion of the universe. Meanwhile gravito-electric charges remain constant for a comoving observer who employs physical coordinates, in a more general scenario the inner product of gravito-electric and gravito-magnetic currents can be thought of as an invariant $I$, which in the case here studied adopts the particularly simple form: $I=Q_{g e} Q_{g m}$, because there are no gravito-magnetic currents outside the mathematical string located on the $\mathbf{z}$-axis. Otherwise we would obtain additional terms related to currents.

\section{Acknowledgements}

J. M. Romero and M. Bellini acknowledge CONICET (Argentina) and UNMdP for financial support.

[1] T. H. R. Skyrme, Proc. Roy. Soc. 262: 233 (1961).

[2] H. B. Nielsen, P. Olesen, Nucl. Phys. B61: 45 (1973).

[3] G. t'Hooft, Nucl. Phys. B79: 276 (1974);

A. M. Polyakov, JETP Lett. 20: 194 (1974).

[4] S. Frolov et al, J. Phys. A47: 085401 (2014).

[5] M. Heydari-Fard, H. Razmi, S. Y. Rokni, Class.Quant.Grav. 30: 165006 (2013).

[6] Ya. B. Zel'dovich, M. Yu. Khlopov, Phys. Lett. B79: 239 (1978).

[7] A. Vilenkin, Phys. Rev. Lett. 46: 1169 (1981); Ibid., Erratum: Phys. Rev. Lett. 46: 1496 (1981).

[8] A. H. Guth, Phys. Rev. D23: 347 (1981);

A. D. Linde. Phys. Lett. B129: 177 (1983).

[9] A. Raya, J. E. Madriz Aguilar, M. Bellini, Phys. Lett. B638: 314(2006);

J. E. Madriz Aguilar, M. Bellini, Phys. Lett. B642: 302 (2006).

[10] J. M. Romero, M. Bellini. Phys. Lett. B674: 143(2009). 
[11] F. A. Membiela, M. Bellini, Phys. Lett. B674: 152(2009);

F. A. Membiela, M. Bellini, Phys. Lett. B685: 1(2010).

[12] P. S. Wesson, Phys. Lett. B276: 299 (1992);

J. M. Overduin and P. S. Wesson, Phys. Rept. 283: 303 (1997);

T. Liko, Space Sci. Rev. 110: 337 (2004).

[13] J. E. Campbell, A course of Differential Geometry (Charendon, Oxford, 1926);

L. Magaard, Zur einbettung riemannscher Raume in Einstein-Raume und konformeuclidische Raume. (PhD Thesis, Kiel, 1963).

[14] S. Rippl, C. Romero, R. Tavakol, Class. Quant. Grav. 12: 2411 (1995).

[15] F. Dahia, C. Romero, J. Math. Phys.43: 5804 (2002).

[16] F. Dahia, C. Romero, Class. Quant. Grav. 22: 5005 (2005).

[17] J. M. Romero, M. Bellini, Eur. Phys. J.C73: 2317(2013).

[18] R. Weitzenböck. Invarintentheorie. Nordhoff, Groningen (1923).

[19] J. M. Romero, M. Bellini, Gravito-magnetic currents in the inflationary universe from WIMT. E-print arXiv: arXiv: 1402.7288.

[20] H. Kleinert. Gauge Fields in condensed matter: Stresses and defects. Vol. 2. World Scientific, Singapur (1989).

[21] A. Vilenkin, E. P. S. Shellard, Cosmic Strings and Other Topological Defects, chapter 14, Eq. (14.1.15). Cambridge U. P. (1994). 